\begin{tabular}{|c|c|c|}
\hline & Int.J.Curr.Microbiol.App.Sci (2016) 5(10): 370-378 & \multirow{2}{*}{ 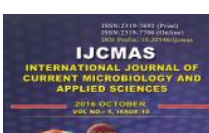 } \\
\hline & \multirow{4}{*}{$\begin{array}{l}\text { International Journal of Current Microbiology and Applied Sciences } \\
\text { ISSN: 2319-7706 Volume } 5 \text { Number } 10(\mathbf{2 0 1 6 )} \text { pp. 370-378 } \\
\text { Journal homepage: } \underline{\text { http://www.ijcmas.com }}\end{array}$} & \\
\hline & & \\
\hline EXCELLENT & & \\
\hline PUBLISHERS & & wwwijijemas com \\
\hline
\end{tabular}

Original Research Article

http://dx.doi.org/10.20546/ijcmas.2016.510.042

\title{
Inducible Clindamycin Resistance among Staphylococcus aureus Isolates in Government Medical College, Aurangabad, India
}

\author{
Shaikh Ambreen Fatema Abdul Hafiz*, M.S. Harbade (Duthade), \\ A.A. Gaikwad, A.S. Damle and J.A. Iravane \\ Department of Microbiology, Government Medical College, Aurangabad, India \\ *Corresponding author
}

Keywords

Clindamycin,

MRSA,

MSSA,

Constitutive

MLSB,

inducible MLSB.

Article Info

Accepted:

14 September 2016

Available Online:

10 October 2016

\section{A B S T R A C T}

Staphylococcus aureus is one of the most common human pathogens with ability to cause wide range of infections. The increasing incidence of a variety of infections due to hospital acquired and community associated methicillin-resistant Staphylococcus aureus (MRSA) has led to emphasis for need of safe and effective agents. Clindamycin is commonly used drug for MRSA as it is safe, effective, less costly and can be given orally. Due to extensive use of this antibiotic, it has developed resistance by this mechanism and hence it is important to detect resistance to clindamycin. The Clinical laboratory Standards Institute (CLSI) recommends D test for detecting inducible resistance phenotypically. Aim of the study was to see the Clindamycin resistance pattern in S.aureus (Staphylococcus aureus) isolates. During a period of one year i.e. in 2015,724 S.aureus isolates from various clinical samples were evaluated and methicillin resistance was determined using Cefoxitin $(30 \mathrm{mcg})$ disc and inducible resistance to clindamycin was detected by D-test as per CLSI guidelines (2014). We observed that among 724 S.aureus isolates inducible resistance was found in 124(17.12\%); 94 (19.02\%) of 494 Methicillin Resistant S.aureus (MRSA) isolates and 30 (13.04\%) of 230 Methicillin Sensitive S.aureus (MSSA) isolates showed inducible resistance. Study showed that $\mathrm{D}$ test should be used as mandatory method in routine disc diffusion testing to detect inducible Clindamycin resistance for optimum treatment of patients.

\section{Introduction}

Staphylococcus aureus is recognized as one of the most common organisms causing nosocomial and community-acquired infections in every region of the world (Yilmaz et al., 2007). Staphylococci are responsible for several suppurative types of infections. They have a differential ability to spread and cause outbreaks in hospitals which is now recognized as an extremely successful human pathogen. Resistance to methicillin emerged shortly after the drug's introduction and is a factor that has helped Staphylococcus aureus become established as a nosocomial pathogen (Barber, 1961). The increasing prevalence of resistance to most antimicrobial agents in staphylococci, 
especially spread of resistant strains in the community, signify the need for new effective agents to treat staphylococcal infections (Lewis et al., 2005). Methicillinresistant Staphylococcus aureus (MRSA)are increasingly being reported as multidrug resistant with high resistance to macrolides (Erythromycin, Clarithromycin) and lincosamides (Clindamycin, Lincomycin) leaving very few therapeutic options (Srinivasan et al., 2002).

Clindamycin resistance in Staphylococcus species can be either constitutive or inducible. The most common mechanism for such resistance is target site modification mediated by erm genes, which can be expressed either constitutive macrolidelincosamide-streptogramin B (constitutive MLSB phenotype) or inducible macrolidelincosamide-streptogramin B (inducible MLSB phenotype). Strains with inducible resistance to clindamycin are difficult to detect in the routine laboratory as they appear Erythromycin-resistant and Clindamycin sensitive in vitro when not placed adjacent to each other. In such cases, in vivo therapy with clindamycin may select constitutive erm mutants leading to clinical therapeutic failure. In case of another mechanism of resistance mediated through msrA genes i.e. efflux of antibiotic, Staphylococcal isolates appear erythromycin-resistant and Clindamycinsensitive both in vivo and in vitro and the strain do not typically become Clindamycin resistant during therapy (Deotale et al., 2010).

Newer antibiotics like vancomycin, linezolid, and quinupristin-dalfopristin have been advocated in the management of such isolates, but recent reports of resistance to these agents raise real concerns over how long these uniform susceptibilities will hold good (Johnson et al., 2003). This has led to renewed interest in the usage of MLSB antibiotics to treat S.aureus infections with, clindamycin being the preferred agent due to its excellent pharmacokinetic properties.

Clindamycin has excellent tissue penetration except for the central nervous system (Sivapalasingam et al., 2010). Macrolide induced Clindamycin resistance was observed among the clinical isolates of Staphylococcus since 1968 which could not be detected by the routine disc diffusion method. From such isolates constitutively resistant mutants are emerged and results in treatment failure with clindamycin in vivo which would be demonstrated on D-test (Frank et al., 2002).

The main aim of this study to inducible clindamycin resistance amongst S.aureus isolates. And to study the utility of D test for the detection of inducible clindamycin resistance in S.aureus isolates.

\section{Material \& Methods}

The study was conducted in the Microbiology Department of Government Medical College Aurangabad during period January 2015to December 2015.Various clinical samples like pus, wound swabs, aspirates, blood, urine, sputum, tracheal aspirate, umbilical cord, catheter tip and body fluids were evaluated and included in our study. Total number of organisms isolated were 3,096. Among the organisms 724 (23.38\%) were Staphylococcus aureus isolates. S.aureus were isolated by using conventional bacteriological methods such as colony morphology, Gram staining, catalase, coagulase test and mannitol fermentation test. All samples were processed as per standard procedures (Baird, 2008). Isolates were subjected to Antibiotic susceptibility testing by Kirby Bauers disk diffusion method on Muller Hinton agar (MHA) according to the Clinical and Laboratory standards Institute (CLSI -2014). 
A 0.5 McFarland suspension of staphylococci was inoculated on Mueller Hinton agar plate, antibiotic discs applied were Gentamicin $(10 \mu \mathrm{g})$, Clindamycin $(2 \mu \mathrm{g})$, Erythromycin $(15 \mu \mathrm{g})$, Tetracycline $(30 \mu \mathrm{g})$, Cotrimaxazole $(25 \mu \mathrm{g})$, Amoxicillin Clavulanate $(30 \mu \mathrm{g})$, Ciprofloxacin $(5 \mu \mathrm{g})$ and Cefoxitin $(30 \mu \mathrm{g})$.

Methicillin resistance was detected by using Cefoxitin disc diffusion. MRSA \& MSSA strains were categorized by phenotypic criteria.494 (68.23\%) were MRSA and 230 $(31.76 \%)$ were MSSA as shown in (Figure $1)$.

D test was also noted on these erythromycin resistant strains for detection of various clindamycin resistance patterns on the same Antibiotic sensitivity plate.

\section{Method for D test}

For detection of inducible Clindamycin resistance (iMLSB), a disk approximation test was performed by placing a Clindamycin disc 15-26 $\mathrm{mm}$ away from the edge of erythromycin discover the MHA which is inoculated with the test organism. Following overnight incubation at $37^{\circ} \mathrm{C}$, Erythromycin diffuses, it induces, resistance to Clindamycin and results in flattening of the Clindamycin zone just next to the Erythromycin disk, making a D shape, which is interpreted D-test positive, whereas complete zone indicates D-test negative.

\section{ATCC Controls used}

Staphylococcus aureus (ATCC 25923) strains.

Staphylococcus aureus (ATCC 43300) MRSA strains

In house strains of Staphylococcus aureus showing D-test positive.
Three different phenotypes were appreciated after testing and interpreted as follows:

Erythromycin and Clindamycin sensitive isolates -Staphylococcal isolates sensitive to both erythromycin (zone size $\geq 23 \mathrm{~mm}$ ) and Clindamycin (zone size $\geq 21 \mathrm{~mm}$ ) were labelled as having this phenotype. (Refer Figure 2)

MS Phenotype - Staphylococcal isolates exhibiting resistance to erythromycin (zone size $\leq 13 \mathrm{~mm}$ ) while sensitive to Clindamycin (zone size $\geq 21 \mathrm{~mm}$ ) and giving circular zone of inhibition around clindamycin was labelled as having this phenotype. (Refer Figure 3)

Constitutive MLSB Phenotype - this phenotype was labelled for those Staphylococcal isolates which showed resistance to both erythromycin (zone size $\leq 13 \mathrm{~mm}$ ) and clindamycin (zone size $\leq 14 \mathrm{~mm}$ ) with circular shape of zone of inhibition if any around clindamycin. (Refer Figure4)

Inducible MLSB Phenotype Staphylococcal isolates showing resistance to erythromycin (zone size $\leq 13 \mathrm{~mm}$ ) while being sensitive to clindamycin (zone size $\geq 21 \mathrm{~mm}$ ) and giving $D$ shaped zone of inhibition around Clindamycin with flattening towards erythromycin disc were labelled as having this phenotype. (Refer Figure 5)

\section{Results and Discussion}

Among 724 Staphylococcus aureus; 494 (68.23\%) were MRSA and 230 (31.76\%) were MSSA. MRSA and MSSA both were showing D test positive (Refer Figure 6 and 7). Total D test positive were $124(17.12 \%)$ of which $94(19.02 \%)$ cases in MRSA and $30(13.04 \%)$ cases in MSSA shows D test positive. Among MRSA and MSSA, D test negative were $149 \quad(30.16 \%)$ and 
41(17.82\%) respectively. Samples showing both Erythromycin and Clindamycin sensitivity were 202(40.89\%) and $144(17.82 \%)$ and 292 (59\%) samples of MRSA were found to be erythromycin resistant and $86(37.4 \%)$ samples of MSSA were found to be erythromycin resistant. Refer (Table.1) and (Figure 8)

Percentage of both inducible and constitutive resistance was found to be higher in methicillin resistant isolates as compared to methicillin sensitive staphylococci strains

Initial susceptibility report:

1. Erythromycin-R\&Clindamycin-R Resistant to Clindamycin

2. Erythromycin-S \&Clindamycin-S Susceptible to Clindamycin

3. Erythromycin-R but Clindamycin-SNeed $D$ test to confirm Clindamycin resistance

D test interpretation:

1. D test Negative-Clindamycin susceptible (efflux mechanism of erythromycin resistance)

2. $\mathrm{D}$ test Positive-Inducible clindamycin resistance (iMLSB)

Report of D test positive was informed to the Physician of the Hospital and they were advised to withhold clindamycin therapy for that patient.

Before initiating the antimicrobial therapy of infected individuals, the antimicrobial susceptibility testing for clinical isolates is performed to avoid indiscriminate usage of antibiotics on trial and error basis. This is particularly important considering the increase of resistance and the emergence of multidrug resistant organisms in Staphylococcus infections. Production of methylase and efflux proteins is the most widespread and important mechanism of resistance among Staphylococci, conferring resistance to MLSB group of antibiotics.

In our study Methicillin resistance was identified in 68\% isolates of Staphylococcus aureus, similar studies as Gupta et al., and Sharma et al., in India. High rate of methicillin resistance is noted among S.aureus isolates in developed nations (Chelae et al., 2009).

Among them inducible Clindamycin resistance (D test positive) and constitive MLSB was found to be more in MRSA than MSSA, this was in concordance with a few studies reported before (Sanchez et al., 1993).

In MRSA and MSSA isolates of Staphylococcus aureus Erythromycin resistance was found in $292(40 \%)$ cases of 494 and $86 \quad(37.4 \%)$ cases of 230respectively.

Drug of choice in treatment of MRSA is Vancomycin, but it has some limitation. In such cases Clindamycin should be considered for the management of skin and serious soft tissue infections that are sensitive to Clindamycin due to good oral absorption and as a follow-up after intravenous therapy as its efficacy is not affected by high bacterial load at the site of infection and dose adjustment is not required even in severe hepatic or renal dysfunction.

It is less expensive than some of the newer agents with good oral absorption, making it an attractive substitute for outpatients therapy (Sanchez et al., 1993). 
Table.1 Showing antibiotic susceptibility pattern of four different Phenotypes in MRSA and MSSA

\section{Table - 1: Distribution of isolates}

\begin{tabular}{|c|l|c|c|}
\hline Sr.no & \multicolumn{1}{|c|}{$\begin{array}{c}\text { Susceptibility pattern (Phenotype)of Erythromycin } \\
\text { and Clindamycin }\end{array}$} & MRSA (\%) & MSSA (\%) \\
\hline 1 & ERY-S, CL-S & $202(40.89)$ & $144(62.60)$ \\
\hline 2 & ERY-R, CL-R (constitutive MLSB) & $49(9.91)$ & $13(5.66)$ \\
\hline 3 & ERY-R, CL-S, D test negative (MS) & $149(30.16)$ & $41(17.82)$ \\
\hline 4 & ERY-R, CL-S, D test positive (inducible MLSB) & $94(19.02)$ & $30(13.04)$ \\
\hline & Total & $494(68.23)$ & $230(31.76)$ \\
\hline
\end{tabular}

Fig.1 Showing percentages of MRSA and MSSA.

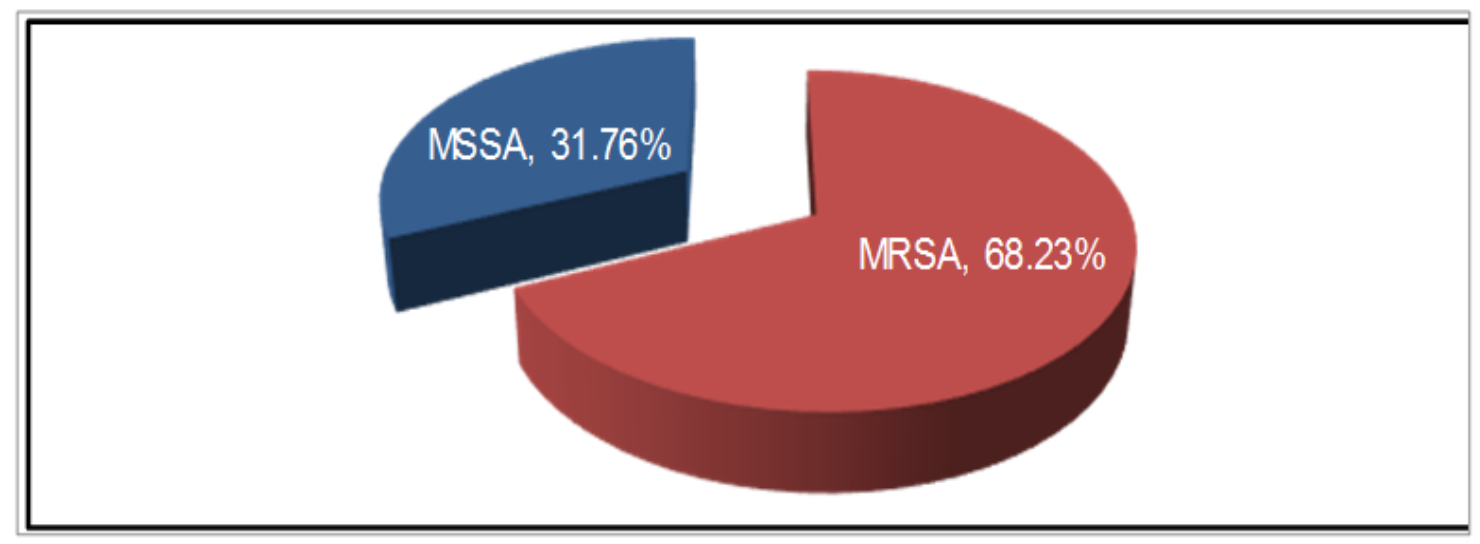

Fig.2 Staphylococcal isolate sensitive to both erythromycin and clindamycin.

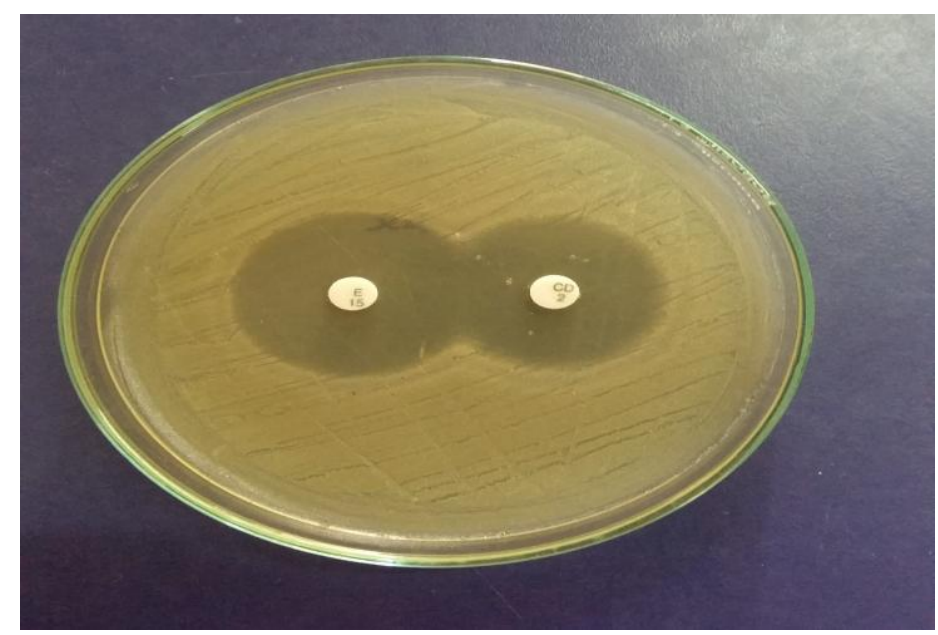


Fig.3 Erythromycin resistant and clindamycin sensitive Staphylococcal isolate. (MS phenotype)

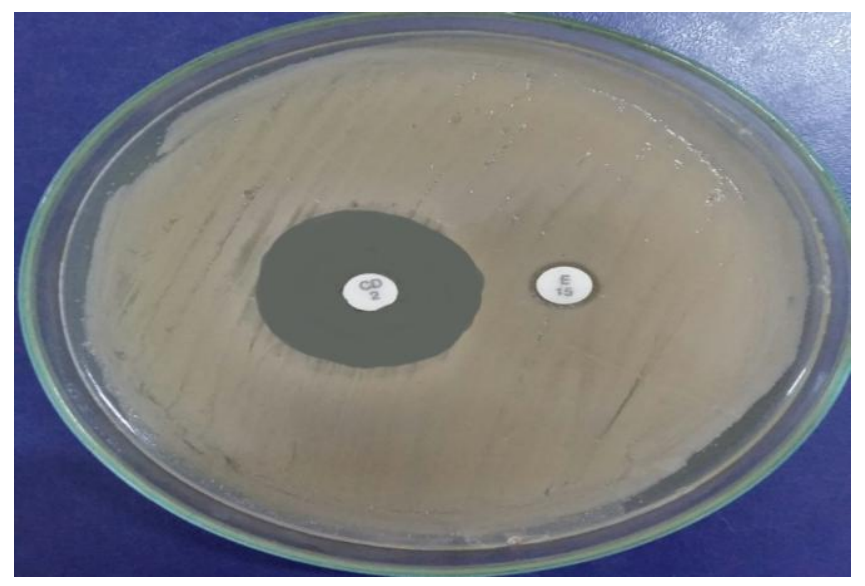

Fig.4 Staphylococcal isolate showing resistant to both erythromycin and clindamycin (Constitutive MLSB phenotype)

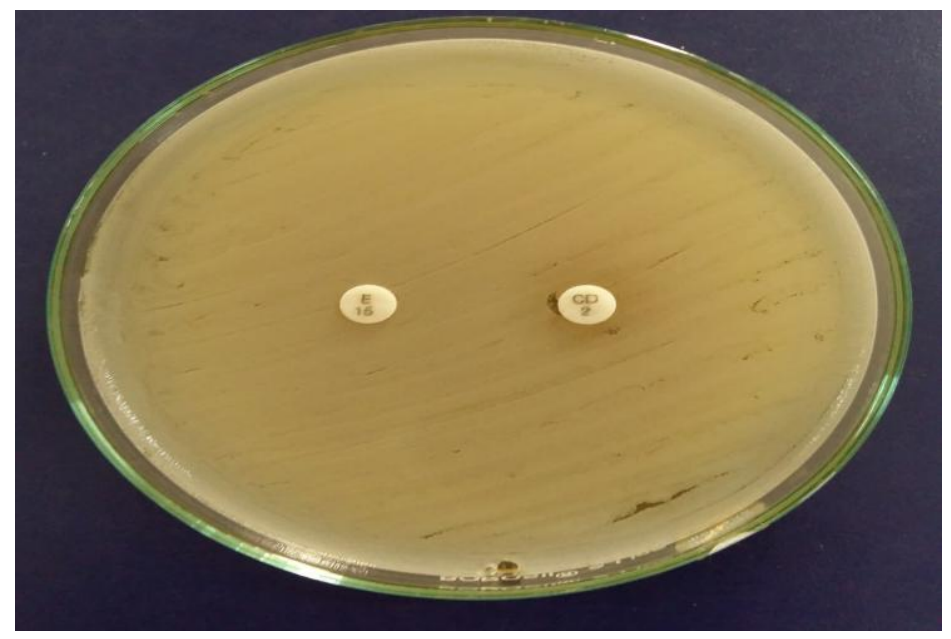

Fig.5 Staphylococcal isolate showing erythromycin resistant and clindamycin sensitive zone, with D shaped zone of inhibition around it with flattening towards erythromycin disc (inducible MLSB phenotype)

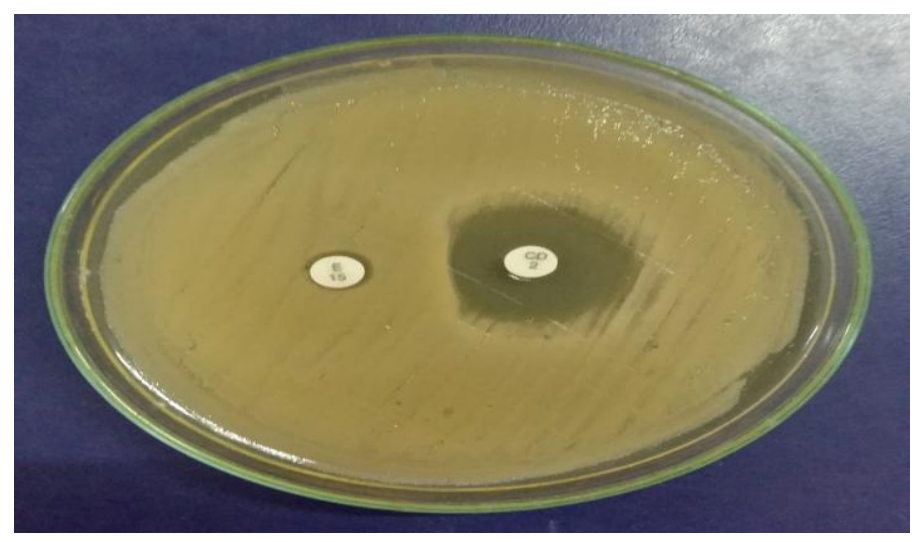


Fig.6 Showing D test positive in MRSA (Methicillin resistance Staphylococcus aureus.

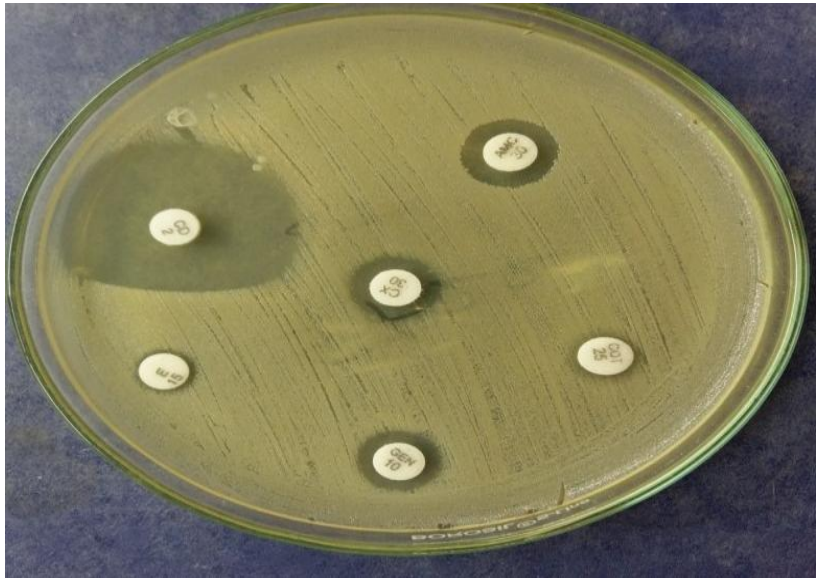

Fig.7 Showing D test positive in MSSA (Methicillin sensitive Staphylococcus aureus).

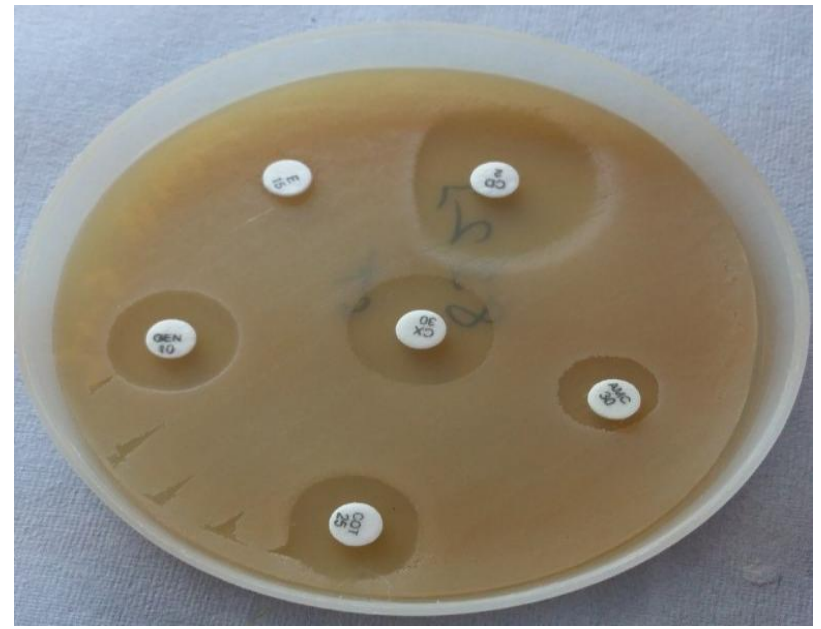

Fig.8 Bar Diagram showing different phenotypes in MRSA and MSSA

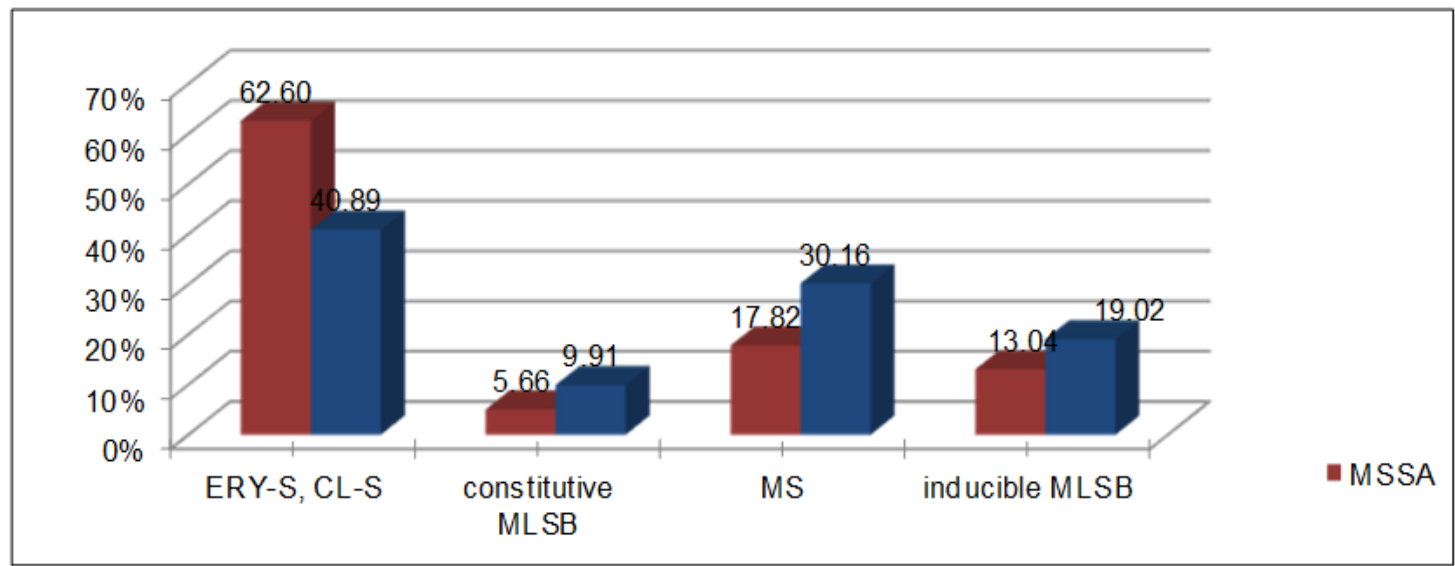


Simple laboratory testing, (i.e. erythromycin, clindamycin'D-zone' test) can separate strains that have the genetic potential to become resistant during therapy from strains that are truly susceptible to clindamycin. Without the double-disk test, all staphylococcal isolates with inducible MLSB would have been mistakenly interpreted as clindamycin-susceptible. On the other side, to categorically consider all erythromycin resistant staphylococci as clindamycin resistant would deny potentially safe and effective therapy for patients infected with isolates that carry only the export mechanism.

In conclusion, in hospital like ours where inducible MLSB strains is found more in both MSSA \& MRSA, we recommend to perform D-test routinely. Inducible clindamycin resistance as noted by a positive $\mathrm{D}$ test should be reported as resistant. A comment should be added that this isolate is presumed to be resistant based on detection of inducible resistance. Clindamycin may still be effective in some patients for empirical outpatient treatment options for staphylococcal infections have become more limited as concerns about the prevalence of MRSA have increased.

Clindamycin should be kept as a reserve drug and be usually advocated in severe MRSA infections depending upon the antimicrobial susceptibility results. Reporting S.aureus as susceptible to clindamycin without checking for inducible resistance may result in institution of inappropriate clindamycin therapy. On the other hand, negative result for inducible clindamycin resistance confirms clindamycin susceptibility and provides a very good therapeutic option.

Use of D test in a routine laboratory enables us in guiding the clinicians in judicious use of Clindamycin, as Clindamycin is not a suitable drug for D test positive MS phenotype resistance was almost equal among isolates; while it can definitely prove to be a drug of choice in case of $D$ test negative isolates.

\section{References}

Baird, D. 2008. "Staphylococcus Micrococcus:Cluster forming Gram positive cocci" In: Collee JG, Fraser AG, Marmion BP, Simmons A, editors, "Mackie and McCartney Practical Medical Microbiology", 14th edition, Edinburg, Churchill Livingstone, pp 245-262.

Barber, M. 1961. Methicillin Resistant Staphylococci. J. Clin. Pathol., 14: 385-93.

Chelae, S., Laohaprertthisarm, V., Phengmak, M., Kongmuang, U., Kalnauwakal, S. 2009. Detection of inducible clindamycin resistance in Staphylococcci by disc diffusion induction test. J. Med. Assoc. Thai., 92(7): 947-51.

Clinical and Laboratory Standard Institute. 2014. Performance standards for antimicrobial susceptibility testing; Twenty-fourth informational supplement. CLSI document M100S24. Wayne, PA: CLSI.

Deotale, V., Mendiratta, D.K., Raut, U., Narang, P. 2010. Inducible clindamycin resistance in Staphylococcus aureus isolated from clinical samples. Indian J. Med. Microbiol., 28(2): 124.

Diekema, D.J., Pfaller, M.A., Schmitz, F.J., Smayevsky, J., Bell, J., Jones, R.N., Beach, M. 2001. Survey of infections due to Staphylococcus species: frequency of occurrence and antimicrobial susceptibility of isolates collected in the United States, Canada, 
Latin America, Europe, and the Western Pacific region for the SENTRY Antimicrobial Surveillance Program, 1997-1999. Clin Infect. Dis., 32(Supplement 2): S114-32.

Eliopoulos, G.M. 2003. Quinupristindalfopristin and linezolid: Evidence and opinion. Clin. Infect. Dis., 36: 473-81.

Frank, A.L., Marcinak, J.F., Mangat, P.D., Tjhio, J.T., Kelkar, S., Schreckenberger, P.C., Quinn, J.P. 2002. Clindamycin treatment of methicillin-resistant Staphylococcus aureus infections in children. The Pediatric Infect. Dis. J., 21(6): 530-4.

Johnson, A.P., Woodford, N. 2002. Glycopeptide-resistant Staphylococcus aureus. J. Atimicrob. Chemother., 50: 621-3.

Lewis, J.S. 2005. 2nd, Jorgensen JH. Inducible clindamycin resistance in staphylococci: Should clinicians and microbiologists be concerned? Clin. Infect. Dis., 40: 280-5.

Sanchez, M.L., Flint, K.K., Jones, R.N. 1993. Occurrence of macrolidelincosamide-streptogramin resistances among staphylococcal clinical isolates at a University Medical Center: Is false susceptibility to new macrolides and clindamycin a contemporary clinical and in vitro testing problem? Diag. Microbiol. Infect. Dis., 16(3): 205-13.

Sharma, N.K., Garg, R., Baliga, S., Bhat, K.G. 2013. Nosocomial infections and drug susceptibility patterns in methicillin sensitive and methicillin resistant Staphylococcus aureus. $J$. Clin. Diagn. Res., 7: 2178-80.

Sivapalasingam, S., Steigbigel, N.H. 2010. Macrolides, clindamycin, and ketolides. Mandell GL, Bennett JE, Dolin R (eds.). Mandell, Douglas, and Bennetts Principles and Practice of Infectious Diseases. 7th ed. Churchill Livingstone: Elsevier, 427-8.

Srinivasan, A., Dick, J.D., Perl, T.M. 2002. Vancomycin resistance in staphylococci. Clin. Microbiol. Rev., 15: 430-8.

Yilmaz, G., Aydin, K., Iskender, S., Caylan, R., Koksal, I. 2007. Detection and prevalence of inducible clindamycin resistance in staphylococci. J. Med. Microbiol., 56(3): 342-5.

\section{How to cite this article:}

Shaikh Ambreen Fatema Abdul Hafiz, M.S. Harbade (Duthade), A.A. Gaikwad, A.S. Damle and J.A. Iravane. Inducible Clindamycin Resistance among Staphylococcus aureus Isolates in Government Medical College, Aurangabad, India. Int.J.Curr.Microbiol.App.Sci. 5(10): 370378. doi: http://dx.doi.org/10.20546/ijcmas.2016.510.042 